Swarthmore College

Works

$11-1-2001$

\title{
Energetic Particles And Magnetohydrodynamic Activity In The Swarthmore Spheromak Experiment
}

G. Qin

Vyacheslav S. Lukin , '00

C. D. Cothran

Michael R. Brown

Swarthmore College,doc@swarthmore.edu

W. H. Matthaeus

Follow this and additional works at: https://works.swarthmore.edu/fac-physics

Part of the Physics Commons

Let us know how access to these works benefits you

\section{Recommended Citation}

G. Qin; Vyacheslav S. Lukin , '00; C. D. Cothran; Michael R. Brown; and W. H. Matthaeus. (2001). "Energetic Particles And Magnetohydrodynamic Activity In The Swarthmore Spheromak Experiment". Physics Of Plasmas. Volume 8, Issue 11. 4816-4825. DOI: 10.1063/1.1405015

https://works.swarthmore.edu/fac-physics/105

This work is brought to you for free by Swarthmore College Libraries' Works. It has been accepted for inclusion in Physics \& Astronomy Faculty Works by an authorized administrator of Works. For more information, please contact myworks@swarthmore.edu. 


\section{AIP | Physics of Plasmas}

\section{Energetic particles and magnetohydrodynamic activity in the Swarthmore Spheromak Experiment}

G. Qin, V. S. Lukin, C. D. Cothran, M. R. Brown, and W. H. Matthaeus

Citation: Physics of Plasmas (1994-present) 8, 4816 (2001); doi: 10.1063/1.1405015

View online: http://dx.doi.org/10.1063/1.1405015

View Table of Contents: http://scitation.aip.org/content/aip/journal/pop/8/11?ver=pdfcov

Published by the AIP Publishing

\section{Articles you may be interested in}

Hard magnetohydrodynamic limit in $1 / 3$ sawtooth like activity in LHD

Phys. Plasmas 21, 032501 (2014); 10.1063/1.4867066

Theory of magnetic field line random walk in noisy reduced magnetohydrodynamic turbulence

Phys. Plasmas 20, 012308 (2013); 10.1063/1.4789606

Three-dimensional magnetohydrodynamics simulations of counter-helicity spheromak merging in the

Swarthmore Spheromak Experiment

Phys. Plasmas 18, 112512 (2011); 10.1063/1.3660533

Energetic particles from three-dimensional magnetic reconnection events in the Swarthmore Spheromak Experiment

Phys. Plasmas 9, 2077 (2002); 10.1063/1.1458589

Numerical modeling of magnetohydrodynamic activity in the Swarthmore Spheromak Experiment

Phys. Plasmas 8, 1600 (2001); 10.1063/1.1362294

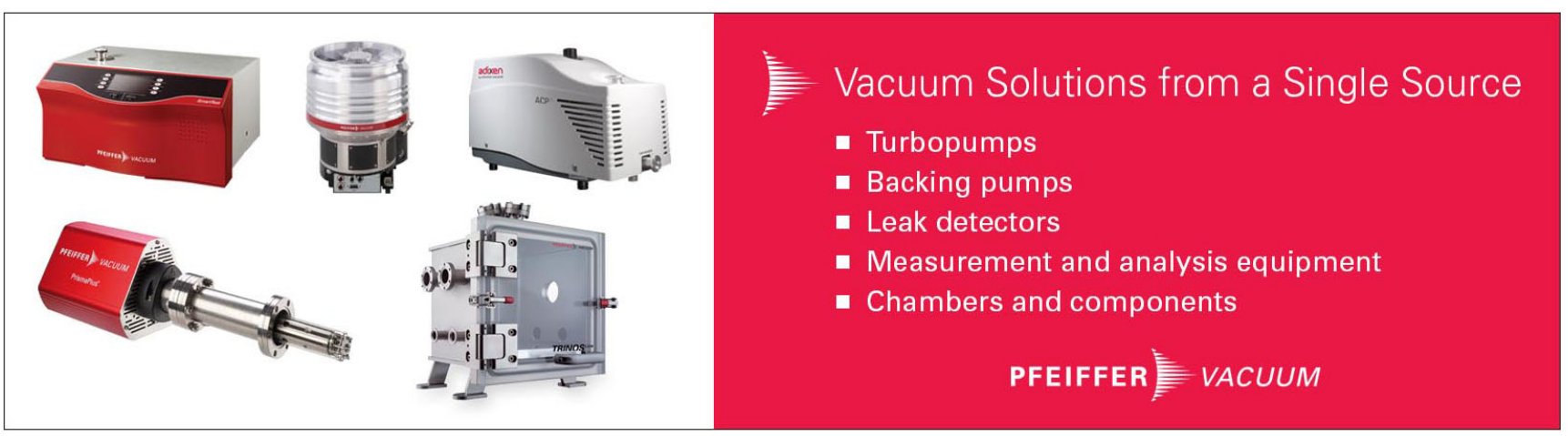




\title{
Energetic particles and magnetohydrodynamic activity in the Swarthmore Spheromak Experiment
}

\author{
G. $\operatorname{Qin}^{\mathrm{a})}$ \\ Bartol Research Institute, University of Delaware, Newark, Delaware 19716
}

V. S. Lukin, C. D. Cothran, and M. R. Brown

Department of Physics and Astronomy, Swarthmore College, Swarthmore, Pennsylvania 19081-1397

W. H. Matthaeus

Bartol Research Institute, University of Delaware, Newark, Delaware 19716

(Received 12 March 2001; accepted 31 July 2001)

\begin{abstract}
Results from the Swarthmore Spheromak Experiment (SSX) [M. R. Brown, Phys. Plasmas 6, 1717 (1999)] indicate that formation and partial merging of two spheromak plasmas can be described well by a magnetohydrodynamic (MHD) picture in which there is substantial evolution towards force free states within each vessel, while reconnection activity, also described reasonably well by MHD, occurs in the region of interaction. MHD simulations [V. S. Lukin et al., Phys. Plasmas 8, 1600 (2001)] support and provide further detail to this interpretation. In the present study, test particle equations are integrated using MHD data from SSX simulations to further understand the energetic particle fluxes that are observed experimentally. The test particle simulation is run with dimensionless parameters similar to the experiment, and particles are permitted to escape when they encounter the simulated SSX boundaries. MHD activity related to reconnection is responsible for accelerating charged particles. The process includes two phases-a strong but short duration direct acceleration in the quasi-steady reconnection electric field, and a weaker longer lived stochastic component associated with turbulence. (C) 2001 American Institute of Physics.
\end{abstract}

[DOI: $10.1063 / 1.1405015$ ]

\section{OVERVIEW}

Magnetohydrodynamic (MHD) plasma motions are probably responsible for many observed properties of the solar atmosphere, the interplanetary medium, and other astrophysical plasmas. Examples are found in solar flares, as well as in Coronal Mass Ejections, and other phenomena observed in the interplanetary medium, including fluctuations and turbulence. These complex processes involve relaxation and evolution of large-scale fields, formation of small scale features such as shocks and current sheets, and magnetic reconnection. Populations of energized charged particles are also commonly found in these same situations. Laboratory experiments have the potential to provide complementary information concerning the interplay between MHD motions and energetic particles that may further our understanding of these astrophysical processes. Several recent studies ${ }^{1-3}$ have described results from the Swarthmore Spheromak Experiment (SSX) and interpreted the observed dynamics mainly in the context of MHD relaxation and magnetic reconnection. Here we further these studies by examining models for the production of energetic particles associated with the reconnection process in SSX.

The Swarthmore Spheromak Experiment ${ }^{4}$ is designed to study magnetic reconnection and particle acceleration due to the controlled, partial merging of two spheromaks (see Fig. 1). The SSX spheromaks are generated by plasma guns away

${ }^{\text {a)} E l e c t r o n i c ~ m a i l: ~ q i n g a n g @ b a r t o l . u d e l . e d u ~}$ from the reconnection zone. ${ }^{1}$ Neutral gas and vacuum magnetic fields are introduced at the guns but only fully ionized plasma and imbedded magnetic flux convect into the reconnection zone. Apart from activity that is expected near the guns, most of the development and interaction of the spheromaks might be described by a MHD formalism. SSX employs a segmented copper boundary at the midplane to allow partial merging as well as relaxation of each spheromak toward its own equilibrium configuration. Early in the discharge we are able to study driven reconnection as the still forming spheromaks merge at the midplane at the Alfvén speed. ${ }^{2}$ Later in the discharge, the two spheromaks separately relax to nearly force-free equilibria ${ }^{1}$ so that we can study spontaneous reconnection. Energetic particles and soft x-rays traverse the field-free, high vacuum gap between flux conservers to various detectors on the midplane.

In a recent paper $^{3}$ we examined MHD activity in SSX in some detail by carrying out MHD simulation in SSX geometry using the TRIM MHD code developed by Schnack and co-workers. ${ }^{5}$ These simulation results were compared to analogous measurements in SSX. The principal results were (1) spheromak formation and partial relaxation to a force free state occurs in both simulation and experiment; (2) the basic timing of the spheromak interactions is gauged by the magnetic energy density computed over a region near the interaction (reconnection) region-this forms a "clock" for the reconnection process which compares well between SSX and TRIM results; (3) reversal of magnetic field and current filamentation occurs in the reconnection zone in both simulation 


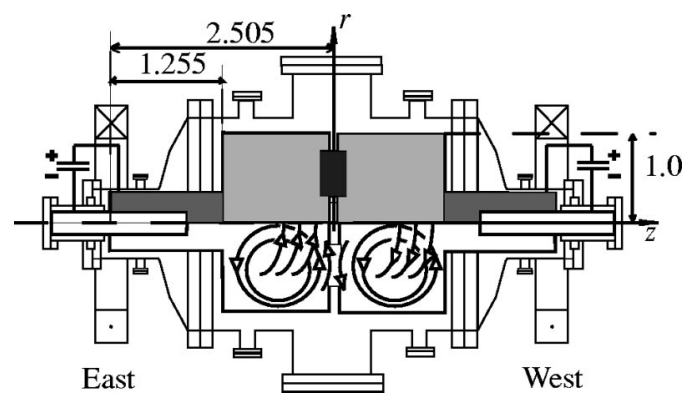

FIG. 1. Computational domain for the TRIM simulation overlaid upon the diagram of SSX. Dimensions are normalized to the flux conserver radius.

and experiment; however, the current sheet thickness is determined by Ohmic dissipation in TRIM and is limited by $c / \omega_{p i}$ in SSX; and (4) Alfvénic outflow, indicative of the reconnection process is observed in both simulation and experiment.

In the present paper we extend this earlier work in two important ways, continuing to compare TRIM results with SSX experimental data. First, we extend analysis of MHD activity in the interaction region between the spheromak plasmas. We examine the small scale magnetic field and the current density using two-dimensional diagnostics. We also look at direct measures of the reconnection rate in TRIM data. Second, we begin here an examination of the processes by which reconnection activity in SSX accelerates charged particles to suprathermal energies. Accurate test particle orbit calculations using TRIM MHD as the applied electromagnetic fields provide the means to efficiently examine the physics of the acceleration process.

We should emphasize at the onset that the MHD model we employ in the TRIM code does not include an accurate description of the local kinetic physics of SSX. The TRIM simulations at present use an isothermal equation of state, and a simple Ohm's law (however, with a fairly accurate Lundquist number), thus ignoring electron pressure, Hall effect and other corrections likely to be of importance in small scale SSX activity. However, our main purpose (see also Ref. 3 ) is to understand the timing, energetics and other gross features of SSX operation. This consists of violent production of plasma by the guns, expansion into the flux conservers, and partial nonlinear relaxation toward a force free state, which is punctuated by intense nonsteady driven reconnection in the interaction zone connecting the two spheromaks regions. Under these circumstances strong inductive electric fields are driven by these energetic large scale plasma processes. Near the interaction region reconnection occurs, and the induced electric field is expected to give way to a resistive component and other corrections, such as a Hall effect electric field, which operate over a small region. These contributions to the electric field within the current channel are driven by the inductive field. Thus, the test particles, responding to the total electric field, may be adequately modeled without a realistic resolution of the kinetic partitioning of the electric field over a region that is expected to be small. On the other hand we are sensitive to the fact that the onset of reconnection, and the structure of the reconnection zone, and the details of the acceleration process may depend on self consistent and kinetic effects which we do not attempt to model here.

Acceleration of charged particles by electric fields associated with reconnection activity is a concept that dates at least to the suggestions of Giovanelli ${ }^{6}$ and Dungey ${ }^{7}$ that strong electric fields may be present near an X-type magnetic neutral point, thus providing a mechanism for acceleration of charged particles in the magnetosphere. Similar arguments were put forth by Sweet $^{8}$ and Parker, ${ }^{9}$ that MHD activity associated with reconnection or merging at field reversal points might accelerate in the vicinity of solar flares. The possibility that reconnection provides a robust acceleration mechanism for charged particles in low density plasmas was one of the original motivations for examining reconnection. This remains one of our motivations for experimental and theoretical study of these processes in SSX. The neutral point acceleration process has been examined earlier in the context of a planar sheet pinch configuration in the presence of finite amplitude fluctuations, ${ }^{10,11}$ and later in strong homogeneous MHD turbulence. ${ }^{12}$ More recently, ${ }^{13}$ acceleration near a three dimensional reconnection zone in the presence of turbulence was examined by Monte Carlo techniques.

In each of the previous studies the reconnection process was found to robustly accelerate particles to high energies. However, in spite of study for half a century in one form or another, this acceleration process is not yet understood completely at either a theoretical or a quantitative level. There is not, for example, a transport model that explains its effects, as there is for diffusive acceleration by shocks (see, for example, Refs. 14-16). In part, this may be because acceleration by reconnection appears to be a complex combination of coherent ("first order") and stochastic ("second order") effects. ${ }^{11}$ At present, the overall importance of acceleration by reconnection in space and astrophysical contexts is incompletely known. It therefore seems particularly germane to gain what insights are available from laboratory studies of this process. The use of the SSX experiment and related simulation studies seem to be appropriate first steps along these lines, as we are unaware of other investigations of this type that have been carried out previously.

The paper is organized as follows. In Sec. II, the methods for the MHD and test particle simulations are presented briefly as well as an outline of the numerical experiments performed. In Sec. III, some new MHD numerical results are presented. Section IV describes test particle simulation results. Discussion and conclusions are given in Sec. V.

\section{MHD AND TEST PARTICLE METHOD}

Here we discuss additional results from TRIM simulations $^{3}$ of SSX. Also, trajectories of charged test particles that respond to the unsteady, inhomogeneous electromagnetic fields from TRIM simulations are computed using a Runge Kutta integration code, using the same boundary description of SSX as was employed in the TRIM simulations. ${ }^{3}$ In this way we can gain insight into the behav- 
ior of suprathermal particle populations in SSX that are only weakly coupled to the thermal MHD plasma by collisions or self consistent electromagnetic fields.

A TRIM ${ }^{5}$ implementation appropriate to SSX was described previously, ${ }^{3}$ along with preliminary MHD results that demonstrate the ability of such simulations to describe, or even reproduce, salient features of SSX dynamics. The MHD model is three-dimensional (3D) cylindrically symmetric single fluid MHD

$$
\begin{aligned}
& \frac{\partial \rho}{\partial t}+\nabla \cdot(\rho \mathbf{u})=0, \\
& \frac{\partial \mathbf{B}}{\partial t}=\nabla \times(\mathbf{u} \times \mathbf{B})+\eta \nabla^{2} \mathbf{B}, \\
& \rho\left[\frac{\partial \mathbf{u}}{\partial t}+(\mathbf{u} \cdot \nabla) \mathbf{u}\right]=\frac{\mathbf{J} \times \mathbf{B}}{c}-\nabla p+\rho \nu \nabla^{2} \mathbf{u} .
\end{aligned}
$$

The simulation code solves for the three components of the vector potential $\mathbf{A}$, the three components of the momentum density $\rho \mathbf{u}$ and the density $\rho$. The magnetic field is $\mathbf{B}$ and, in the MHD approximation, the electric current density is $\mathbf{J}=c \nabla \times \mathbf{B} / 4 \pi$. In the above equations the symbols $\eta$ and $\nu$ represent the resistivity and kinematic viscosity, respectively. For the present simulation, $S \equiv \lambda v_{A} / \eta \approx 1000$ and $R$ $\equiv \lambda v_{A} / \nu \approx 1000$ for characteristic length $\lambda$ and characteristic Alfvén speed $v_{A}$. The parameters and set up of the code are described further in Ref. 3. The code employs an unstructured triangular grid in the $\hat{\mathbf{r}}-\hat{\mathbf{z}}$ plane. Cylindrically symmetric no slip perfectly conducting boundaries are imposed. The startup of TRIM emulates the SSX plasma guns (see Ref. 3) which are used to produce two spheromak plasmas. These interact along slots between the two halves of SSX. The dynamical activity in this interaction region (see Fig. 1) is of central interest in the present paper.

The motion of charged test particles with mass $m$ and velocity $\mathbf{v}$, is governed by the Newton-Lorentz equation

$$
\frac{\mathrm{d}}{\mathrm{d} t}[\gamma m \mathbf{v}(t)]=q\left[\mathbf{E}(\mathbf{x}, t)+\frac{\mathbf{v}(t) \times \mathbf{B}(\mathbf{x}, t)}{c}\right],
$$

where $\gamma$ is the Lorentz factor. To simplify the computations we recognize that energetic particles in SSX are nonrelativistic $(\gamma \rightarrow 1)$ and adopt a normalization in which the unit of speed is the mean Alfvén speed $v_{A}, \lambda$ is a length scale associated with the SSX device (major radius) and the time scale $\tau_{A}$ is the transit time for an Alfvén wave to cross the major radius. As is customary in MHD turbulence calculations, we express the magnetic field in Alfvén speed units. This is accomplished, starting with the magnetic field in laboratory units $\mathbf{B}_{\text {lab }}$ and defining $\mathbf{B}=\mathbf{B}_{\text {lab }} / \sqrt{4 \pi \rho_{0}}$, where $\rho_{0}$ is a characteristic density. We adopt a scale for this magnetic field, $B_{0}$ (equivalent to the characteristic Alfvén speed $v_{A}$ ). All velocities are expressed in units of $v_{A}$. From the equations of motion it follows that the natural scale for electric field becomes $E_{0}=v_{A} B_{0} / c$. In our calculations ${ }^{3}$ we take $v_{A}=7.71 \times 10^{6} \mathrm{~cm} / \mathrm{s}, \lambda=25.4 \mathrm{~cm}$, and $\rho_{0}=2 \times 10^{14}$ proton masses $\mathrm{cm}^{-3}$, so that the Alfvén crossing time is $\tau_{A}$ $=3.24 \mu \mathrm{s}$. The particle equation of motion becomes

$$
\frac{d}{d t} \mathbf{v}(t)=\Omega \tau_{A}[\mathbf{E}(\mathbf{x}, t)+\mathbf{v}(t) \times \mathbf{B}(\mathbf{x}, t)],
$$

where the characteristic particle gyrofrequency is $\Omega$ $=e B_{0} / m c$ for particle mass $m$. The magnetic field $\mathbf{B}(\mathbf{x}, t)$ and electric field $\mathbf{E}(\mathbf{x}, t)$ are obtained from the results of the TRIM simulation, and are now expressed in Alfvén speed units.

The parameter $\Omega \tau_{A} \equiv \alpha$, which arises in (5) as a consequence of our choice of normalizations (see Ref. 11), has a number of important physical interpretations, as can be seen from the relations

$$
\alpha \equiv \Omega \tau_{A}=\Omega \frac{\lambda}{v_{A}}=\left|\frac{q}{q_{i}} \frac{m_{i}}{m}\right| \frac{\lambda}{\left(c / \omega_{p i}\right)}=\frac{\tau_{A}}{\tau_{g}} .
$$

Here, $\tau_{g}=2 \pi / \Omega$ is the test particle gyroperiod, $c / \omega_{p i}$ is the ion inertial length in the background plasma, and $\omega_{p i}$ $=\left(4 \pi n_{0 i} q_{i}^{2} / m_{i}\right)^{1 / 2}$ is the ion plasma frequency in the background plasma, where $q_{i}$ and $m_{i}$ are, respectively, the charge and mass of a background ion. The parameter $\alpha$ couples the particle units to the MHD field units. The later two expressions in Eq. (6) are particularly revealing, as they indicate that $\alpha$ represents the ratio of the relevant MHD and particle time scales $\tau_{g}$ and $\tau_{A}$, or equivalently, the ratio of the two relevant length scales $c / \omega_{p i}$ and $\lambda$. For SSX, $\alpha \approx 15$. In space and astrophysical plasma situations, typically $\alpha$ is much larger (see, for example, Ref. 17).

\section{MHD RESULTS}

An illustration of the SSX device is shown in Fig. 1. Most of our discussion will focus here upon the activity near the central shaded region in which reconnection between the two spheromaks occurs. As the two spheromaks approach one another and begin to interact, there is a buildup of magnetic energy in the interaction region. The time at which this reconnection zone energy reaches its peak has been used earlier as the arbitrary zero for the timing of subsequent interactions. We will also adopt this convention here. Details of SSX dynamics are found in the Refs. 1-3, as are comparisons with TRIM simulation at similar MHD parameters. ${ }^{3}$

We emphasize that we do not attempt a precise numerical simulation of SSX dynamics. This is not feasible at the present time for a number of reasons. For example, there are operational limitations of both SSX and TRIM to certain windows of parameters space. SSX physics is also not expected to be MHD at small scales, as we emphasized in our earlier paper (see Ref. 3). Instead our approach is to try to find dynamical similarities (and differences) between the two cases, so that we may further our understanding of the physics of both systems, and others to which similar ideas apply.

The most obvious signature of reconnection and one that sets up the geometry for neutral point acceleration, is the dynamical appearance of X-point magnetic configurations. This happens in SSX and TRIM in the interaction regions. First appearance is somewhat before the energy maximum at $t=0$, and noticeable X-points persist for several tens of $\mu \mathrm{s}$ thereafter. Figure 2 illustrates X-points in the interaction region both in TRIM simulations and from SSX data. Simula- 

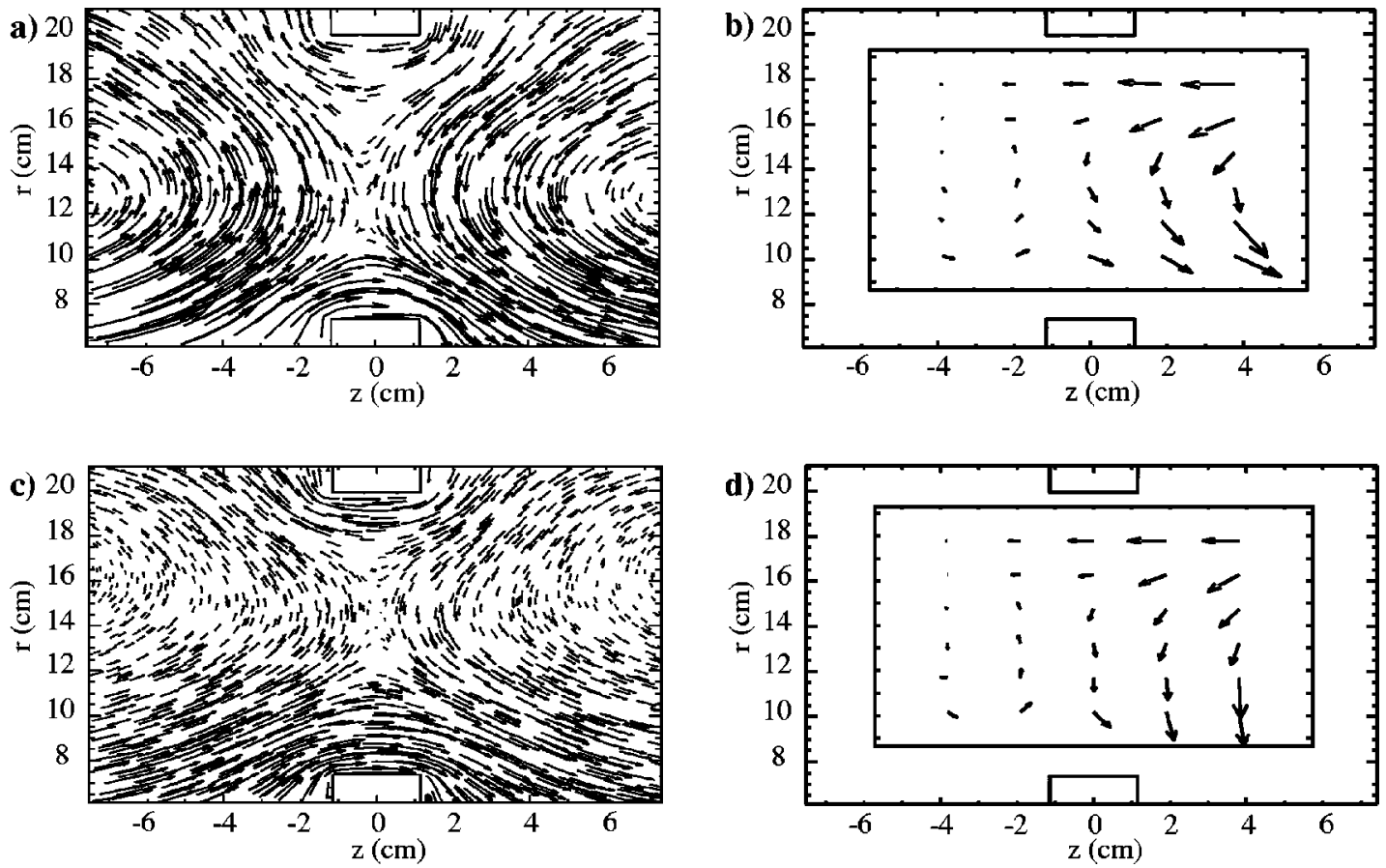

FIG. 2. Magnetic-field lines in the reconnection zone from TRIM simulations [panels (a) and (c)] and from SSX experimental data [panels (b) and (d)]. The SSX data shown are chosen to correspond in time to the TRIM simulation results: (a) time $=3.15 \mu \mathrm{s}$; (b) time $=3.3 \mu \mathrm{s}$; (c) time $=11.73 \mu \mathrm{s}$; (d) time $=11.6 \mu \mathrm{s}$. The plots show X-point formation in the reconnection zone for both simulation and experimental data.

tion times of $t=3.15 \mu \mathrm{s}$ and $t=11.73 \mu \mathrm{s}$ are chosen from TRIM, and SSX data are selected at approximately the same time after the energy maximum for the discharge. Note that the Alfvén speed in the TRIM simulation is approximately $10 \mathrm{~cm} / \mu \mathrm{s}$; more precisely it corresponds to a $3.24 \mu$ s Alfvén crossing time of the $25.4 \mathrm{~cm}$ major radius of the device.

The reader should take note of the relatively greater degree of symmetry in the TRIM X-points compared to the SSX X-points. This is fundamental only in that it points out the highly fluctuating and irregular nature of SSX dynamics. Slight asymmetries in the functioning of the plasma guns can induce significant irregularity in the encounter of the plasmoids when they arrive at the interaction region. Similar irregularities can be seen in TRIM in some runs, such as when mesh differences between the east and west guns induce small asymmetries that grow in time (not shown). The important similarities between SSX and TRIM data in Fig. 2 are related to the highly dynamic driven reconnection that is induced in each case by the encounter of the two spheromaks.

Figure 3 shows the azimuthal component of electric current density $J_{\phi}=\partial_{z} B_{r}-\partial_{r} B_{z}$ from both TRIM data and SSX data for the same cases and at the same times as those shown in Fig. 2. This quantity is determined for SSX by computing the local circulation density around points on a twodimensional lattice of magnetic field probes, described in more detail by Kornack et $a l^{2}$ In each case, one can see that the electric current density is concentrated into filament-like structures near the center of the interaction region. This is associated with the strong shear seen in the magnetic field in the X-point regions. This is a classical signature of reconnection. Also in both experiment and simulation, we can see that the current in regions just beyond the central filament is systematically of a sign opposite to that of the central current channel. This corresponds to flux bunching near the reconnection site and is a familiar feature of highly nonlinear reconnection seen in simulations. We are not aware that this has been reported previously from experiments.

As current filamentation is a signature of the reconnection process, so is the production of mean square electric current density. This provides a global or volume averaged measure of filamentation, and also provides a measure of the production of small scale magnetic structure. In MHD, the production of small scales can indicate strong nonlinearity or even a cascade in steady state turbulence. Here we choose the region of interest to be demarcated by a rectangular box (in the $r-z$ domain) centered in the interaction zone, defined by: $r=(0.4,0.7)$ and $z=(-0.15,0.15)$, in normalized units. Figure 4 shows the mean square current density vs. time for the TRIM simulation. This quantity does not compare very well in its details with SSX data apart from gross features such as the peak near $t=0$. Such agreement would not be expected since the current sheet thinning in SSX is established by kinetic Hall effects ${ }^{3}$ and not by a simple Ohm's law, as in the present implementation of the TRIM MHD simulations. However, the electric field associated with the reconnection process (and therefore the reconnection rate) are expected in both cases to be controlled by the large-scale dynamic of the encounter between the two spheromaks. Differences in detailed current sheet structure clearly should depend upon the details such as inclusion of generalized Ohm's law terms such as the Hall effect. Presumably this would give a more correct local structure in comparison with SSX, but it may not change significantly any process that is prin- 

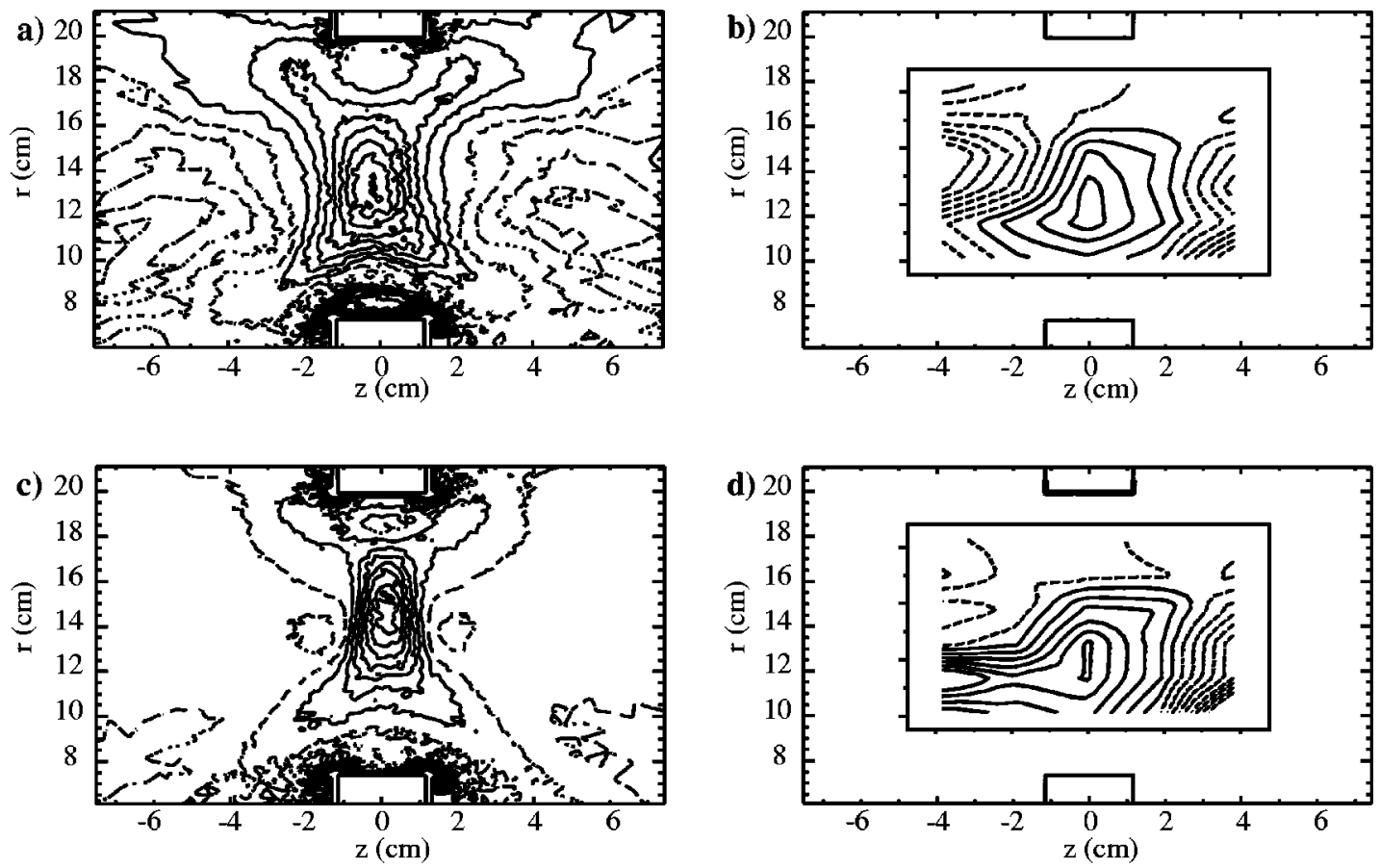

FIG. 3. Contour plots of $J_{\phi}$ computed from the curl of the magnetic-field data in the TRIM simulations are shown in panels (a) and (c) and the corresponding experimental data from SSX is shown in panels (b) and (d). The data correspond to that shown in Fig. 2: (a) time $=3.15 \mu \mathrm{s}$; (b) time $=3.3 \mu \mathrm{s}$; (c) time $=11.73 \mu \mathrm{s} ;$ (d) time $=11.6 \mu \mathrm{s}$.

cipally regulated by large-scale dynamics. However the present treatment could be improved by including more realistic kinetic effects in the current sheet region, a more complete equation of state, and self consistent effects of the energized particle population. Each of these might enable better correspondence to be drawn between simulation and the experimental results. Specifically, with regard to Fig. 4, the reconnection process appears to begin earlier in SSX than in TRIM, presumably due to kinetic processes, and the subsequent burst of mean-square current activity is more intense in

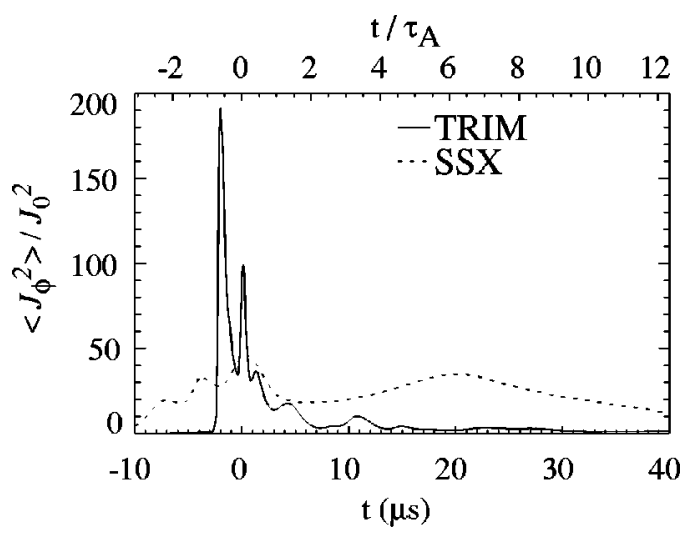

FIG. 4. Shown is the mean-square $\phi$-component of the MHD current $\left\langle J_{\phi}^{2}\right\rangle / J_{0}^{2}$ computed from the reconnection zone in the simulation, as a function of time. The 2D volume of integration is defined by: $r=(0.4,0.7)$ and $z=(-0.15,0.15)$, in normalized units. Large mean-square current in the reconnection zone is indicative of intense turbulence and/or reconnection in this region. Note that, following the period of very intense oscillatory activity around $t=0 \mu \mathrm{s}$, there is a secondary peak near $t=11 \mu \mathrm{s}$. This corresponds to one of the times of Figs. 2 and 3. Also shown is the same quantity from SSX 2D magnetic probe data. See discussion in the text. the purely resistive TRIM simulation. Requisite improvements in modeling remain to be investigated in future, more complete SSX simulation studies.

In Fig. 4, $t=0$ corresponds again to the time of maximum magnetic energy in the reconnection zone. For TRIM results there are there are two very large pulses of mean square current, both near $t=0$, corresponding to strong, driven response of the current to the collision of the spheromaks. It appears that the initial interaction is strongly driven by the inertia of the plasmoids as they collide. There is also unsteady but generally declining activity in the mean-square current for a number of Alfvén crossing times thereafter. A similar diagnostic of mean-square current computed from the 2D SSX magnetic probe array is included in Fig. 4. Although the timings are roughly similar, we note that the levels are much lower in SSX than in TRIM. This may be due to differences in the Ohm's law. However the measured SSX current channel may be limited by the coarse resolution of the probe array. Other diagnostics (not shown) similar to Figs. 2 and 4 indicate that magnetic $\mathrm{X}$-points, current concentrations and other indicators of reconnection persist through that period. However, the reconnection at these later times seems to be much better characterized as spontaneous, and not strongly driven by the plasmoid motions, as the spheromaks have settled down into their formation phase at these later times (see, for example, Refs. 1 and 3).

Another quantity of great interest with regard to reconnection activity is illustrated in Fig. 5. This quantity is the strength of the induced electric field averaged over the interaction region, and normalized by the product of the characteristic Alfvén speed and the characteristic magnetic field strength in the SSX/TRIM domain. In a steady reconnection 


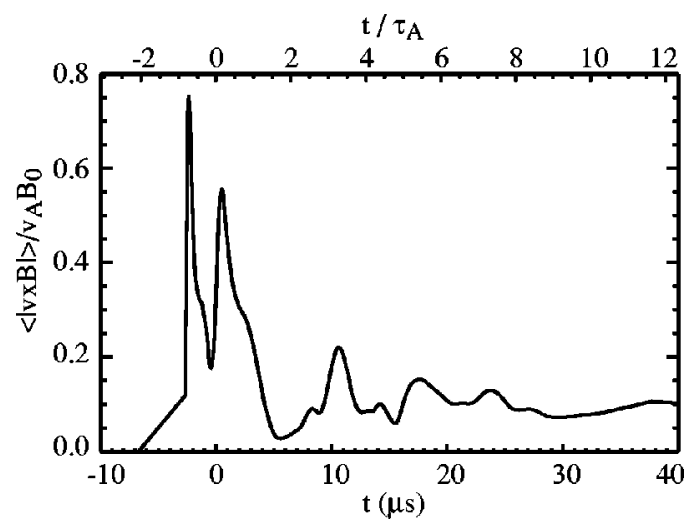

FIG. 5. Plotted is a diagnostic of the reconnection rate in the reconnection region, the induced electric field $\langle|\mathbf{v} \times \mathbf{B}|\rangle /\left(v_{A} B_{0}\right)$ vs time, where $v_{A}$ $=\left\langle\sqrt{B(t)^{2} /(8 \pi \rho(t))}\right\rangle$ is time variable. The two-dimensional (2D) volume of integration is, as in Fig. 4, defined by: $r=(0.4,0.7)$ and $z$ $=(-0.15,0.15)$, in normalized units.

model, this quantity would be uniform in space. The induced electric field normalized in this way may be thought of as the fraction of the Alfvén speed at which plasma can approach the reconnection zone. ${ }^{18}$ Inflowing plasma carries the magnetic field which is to be reconnected, and then the plasma and flux are expelled into the regions that may be referred to as the reconnected islands. Thus the normalized induced electric field is a natural measure of the rate of magnetic reconnection. We can see from the Figure that there are pulses of strong induced electric field, which we associate with stronger reconnection, in the same time periods in which we saw strong current filamentation in Fig. 4. The very high values of the reconnection rate $\sim 0.7$ in these pulses again supports the interpretation that the reconnection during these times is strongly driven. Subsequently, the normalized rate of reconnection fluctuates between values of about 0.05 to 0.2 with an average of about 0.1 from $t$ $=5 \mu \mathrm{s}$ to the end of the data record at $t=40 \mu \mathrm{s}$. This is a very interesting number because it has been cited empirically as a "standard" reconnection rate, first in turbulent MHD reconnection at high Reynolds numbers, ${ }^{10}$ and second in collisionless reconnection as seen in hybrid ${ }^{19}$ and Hall $\mathrm{MHD}^{20,21}$ simulations. However, a full theoretical understanding of the reconnection rate is yet to be presented.

\section{TEST PARTICLE RESULTS}

Numerical experiments with test particle trajectories were carried out using the TRIM data. Two types of calculations were done. In the first kind, a large number of test particles, typically $N_{p}=10^{5}$ protons, are injected into the interaction region with random positions and gyrophases, but all with speed $v_{A}$. The integration of the test particle orbits commences at $t \approx-3 \mu \mathrm{s}$ of the TRIM simulation, about 1 Alfvén time before the local magnetic energy peak in the reconnection zone. Subsequently, if a particle encounters the wall of the simulated SSX device, its orbit calculation is halted and it is considered to have "escaped." Particles are lost through escape in this type of calculation and are not replaced. In the second type of simulation, when a particle
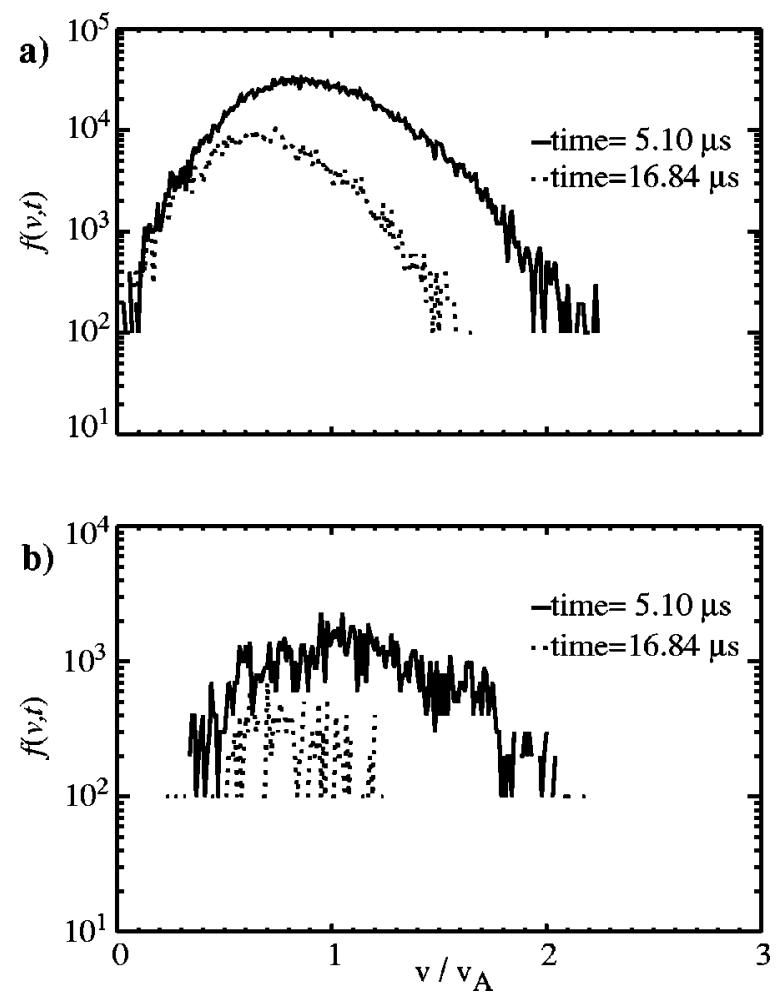

FIG. 6. "Tail formation." Distribution functions $f(v, t)$ of particles remaining within the simulation domain (top panel), and of particles that escape near a given time during a time interval of $\delta t=1.62 \mu \mathrm{s}$ (bottom panel) for two times $t=5.10 \mu \mathrm{s}$ and $t=16.84 \mu \mathrm{s}$. Note that, though statistics for the bottom panel is lower, a flatter distribution of the escaping particles is apparent. In all of the simulation, $\alpha=15.56$.

escapes, it is replaced with another particle in the interaction zone, having the same position as the original particle and also having speed $v_{A}$. This is called a "resupply" run and the total particle number within the SSX domain is in this case, $N_{p}=10^{4}$, a constant. For all test particle simulations discussed in this paper, the coupling parameter that matches particle to MHD units is the typical SSX value $\alpha=\Omega \lambda / v_{A}$ $=15.56$.

An overall view of the energized particle distribution is shown in Fig. 6 for a run with no resupply of particles. The top panel shows distributions in speed of particles that remain within the confines of SSX, for two times. One can readily see that particles have been lost by the later time $t$ $=16.84 \mu \mathrm{s}$, as the distribution at the earlier time $t$ $=5.10 \mu \mathrm{s}$ has distinctly more area under the curve. Keeping in mind that each particle had initial speed of $1 v_{A}$, the distributions become broadened substantially, with significant numbers of particles evolving both to higher and to lower energies. The peak of the distribution remains near the initial speed at $t=5.10 \mu \mathrm{s}$, but by $t=16.84 \mu \mathrm{s}$ the most probable speed has noticeably decreased.

The bottom panel of Fig. 6 shows distributions of particles that have escaped from the SSX/TRIM simulation during an interval of time of duration $1.62 \mu$ s centered about the same time as in the top panel, $t=5.10 \mu \mathrm{s}$ and $t=16.84 \mu \mathrm{s}$. It is evident that the distribution of escaping particles is harder (flatter) than the distribution of particles remaining in the 


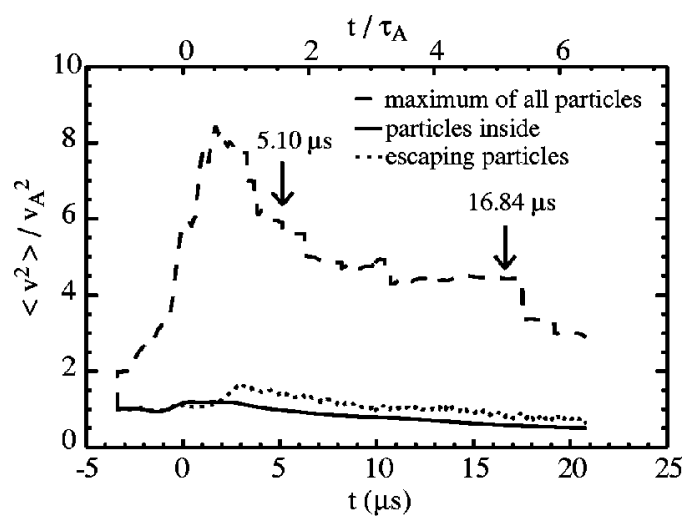

FIG. 7. Time history of three quantities that indicate energization of particles: Top trace (dashed line) is the maximum $v^{2}$ of all particles at a given time; solid line is the mean square velocity $\left\langle v^{2}(t)\right\rangle$ of all particles within the simulation domain, and the dotted line is $\left\langle v^{2}(t)\right\rangle$ for all particles escaping in a window of time (width $\delta t=1.62 \mu \mathrm{s}$ ) centered at time $t$.

simulation domain. One concludes that more energetic particles are more likely to escape by encountering the walls.

Another view of the dynamics of the particle distributions is provided in Fig. 7, which shows maximum and mean-square velocities vs time. The top trace shows maximum $v^{2}$ computed over all the particles within the simulation domain at a given time. The peak in this curve, near $t$ $=2-3 \mu \mathrm{s}$, occurs about an Alfvén transit time after the period of greatest activity in the induced electric field, meansquare current and magnetic energy that occurs around $t$ $=0 \mu \mathrm{s}$. The peak lasts for about an Alfvén time, as did the period of most intense activity in the underlying MHD quantities. All of this is consistent with the picture ${ }^{11,22}$ that the turbulent neutral point acceleration mechanism attains maximum particle energies on a time scale of the order of the Alfvén time. After this peak there is a broader plateau of maximum $v^{2}$ lasting for several Alfvén crossing times. The acceleration process is still active during this period of spontaneous or weakly driven reconnection. The two bottom traces show the mean square particle velocity $\left\langle v^{2}(t)\right\rangle$ vs time of the particles within SSX/TRIM, and also for particles escaping near time $t$. Now we can see that the distribution is the broadest during the high activity period just after $t$ $=0 \mu \mathrm{s}$, and that this "temperature" decreases thereafter as the reconnection and MHD activity decay in time. The escaping particles have a mean-square velocity that peaks a little later, and which remains above the value for the internal population for all later times. Clearly, it is the faster particles that tend to escape more easily.

The distribution functions give us a good idea about the collective behavior of the particles, but they do not tell us very completely how individual particles respond to the MHD electric fields. To uncover more about this issue, we compute the mean-square velocity increment versus time of separation. That is, for each particle we compute the square of the change in its toroidal velocity for a specified time separation $\tau$, that is $\Delta v_{r}(t, \tau)^{2}+\Delta v_{\phi}(t, \tau)^{2}$, where $\Delta v_{i}(t, \tau)=v_{i}(t+\tau)-v_{i}(t)$. We average this over origin in time $t$ and over particles. The result is a mean-square toroidal velocity increment, a function only of time separation $\tau$. We

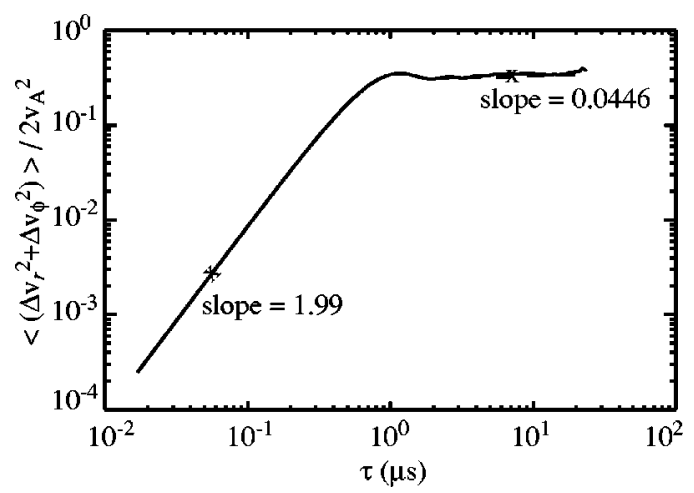

FIG. 8. Mean square velocity increment, $\left\langle\left(\Delta v_{r}^{2}+\triangle v_{\phi}^{2}\right)\right\rangle / 2 v_{A}^{2}$ vs time in LOG-LOG plot, as defined in the text. Note that for the separation time of less than $1 \mu \mathrm{s}$, the slope of the curve is near 2.0 .

normalize to $2 v_{A}^{2}$. Figure 8 shows the result of this computation for the same TRIM/test particle simulation shown in Figs. 6 and 7. The short time response of the particle is distinctive-we see that for time increments less than about $1 \mu \mathrm{s}$, the velocity increments scale like $\tau^{1.99}$. This is exceedingly close to the $\tau^{2}$ dependence that would be expected for particles accelerated in a constant electric field. It is highly suggestive to interpret this as follows: A typical particle finding itself in or near the interaction zone between the spheromaks is likely to experience acceleration associated with the MHD induced electric field, which is approximately the electric field associated with the reconnection process. However, the time of exposure of the particle to this electric field is relatively short-a fraction, perhaps $1 / 3$, of an Alfvén transit time. For time increments longer than $1 \mu \mathrm{s}$, it is unlikely that the particle will continue to experience the effects of this coherent electric field. Instead the velocity changes are random, and in this case sub-diffusive, in that the velocity increments increase slower than linearly in time. The first part of this described scenario is quite similar to the coherent component of acceleration by turbulent reconnection described by Ambrosiano et al. ${ }^{11}$ In the present case, the time of exposure to the coherent electric field is probably determined by a combination of three factors: The time dependence of the electric field, the trajectory of the particle leaving the interaction/reconnection zone, and the possibility that the particle escapes the SSX boundaries. The lack of a truly diffusive spread of velocities beyond time increments of $1 \mu \mathrm{s}$ is probably due to the fact that the correlation length of the fluctuation is comparable to the size of the system. Real diffusion, with $\left\langle(\Delta \mathbf{v})^{2}\right\rangle \propto \tau$, requires that the forces experienced by each particle average to zero, thus that the fluctuations as observed by the particles average to zero. This is expected only if the particle trajectories span more than a correlation length of the fluctuations. We note also that the coherent velocity changes at short time are composed of a mixture of accelerating and decelerating particles, which is indicated by spread of the particle distributions to both higher and lower speeds.

As we have noted, in the test particle simulations with no resupply of particles, the number of particles remaining within the simulation domain decreases in time, as particles, 


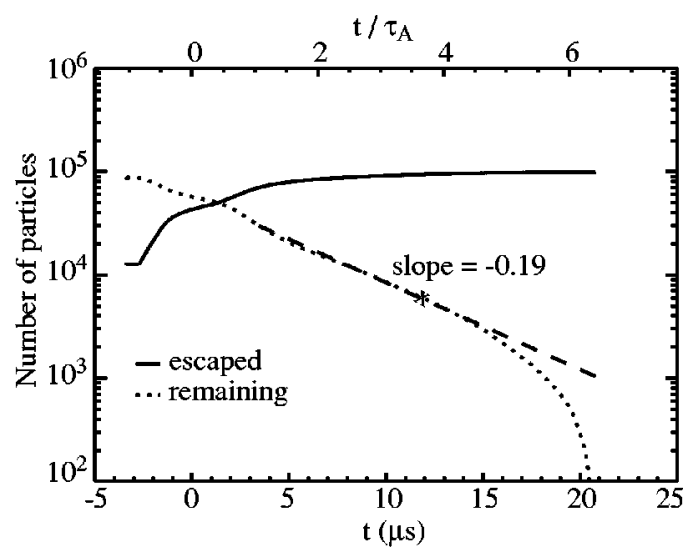

FIG. 9. Plot of the number $N(t)$ of particles remaining within the simulation domain and those escaping the domain vs time. Note that the escape is well approximated by an exponential $e^{-t / \tau}$, corresponding to characteristic escape time of $\tau=1 / 0.19 \approx 5 \mu \mathrm{s}$.

especially those that have been accelerated, encounter the wall and are lost. This is quantified in Fig. 9, which shows the number of particles remaining within the SSX/simulation domain vs time, for an $N_{p}=10^{5}$, no-resupply case. Also shown is the complementary quantity-the number that have escaped vs. time. It can be seen that the number of particles remaining within the simulation domain decrease approximately as an exponential $N(t) \sim e^{-t / 5 \mu \mathrm{s}}$ for times up to around $t=15 \mu \mathrm{s}$. Note that the macroscopic resistive decay time for SSX is around $100 \mu \mathrm{s} .^{1,2}$

We turn now to a brief discussion of test particle simulations in which particles are resupplied. When a particle encounters the boundary, its position and velocity cease evolving - it "escapes" from the SSX boundaries. We replace it with a new particle moving at the Alfvén speed and positioned within the interaction region. This allows a constant number of particles to be maintained in the sample of dynamically evolving trajectories. However, one should not interpret diagnostics based on the resupply strategy as corresponding to steady state statistical distributions. The reason is, of course, that SSX and the TRIM/SSX simulation are not in a steady state. So, the main reason for adopting the resupply strategy is to maintain better statistics for the test particles at later times.

Distributions of particles in speed for a particle resupply run are shown in Fig. 10, for a relatively early time $t$ $=0.6 \mu \mathrm{s}$, and for a time well into the formation phase, $t$ $=15.6 \mu \mathrm{s}$. The top panel shows the distribution function $f(v, t)$ of particles remaining within the simulation domain. Here, the distribution at the earlier time is much broader. This is during the highly active period when the reconnection is rapid and driven. There is, at both times, a core distribution centered about $1 v_{A}$, which is expected since that is the speed at which particles are injected. In the core, the particles are not far from a Maxwellian distribution, but only for speeds very close to $1 v_{A}$. At higher, and at lower speeds, the distribution resembles an exponential.

In the bottom panel of Fig. 10, the speed distribution of test particles that escape the SSX domain is examined. This addresses the question: What kind of particle distributions
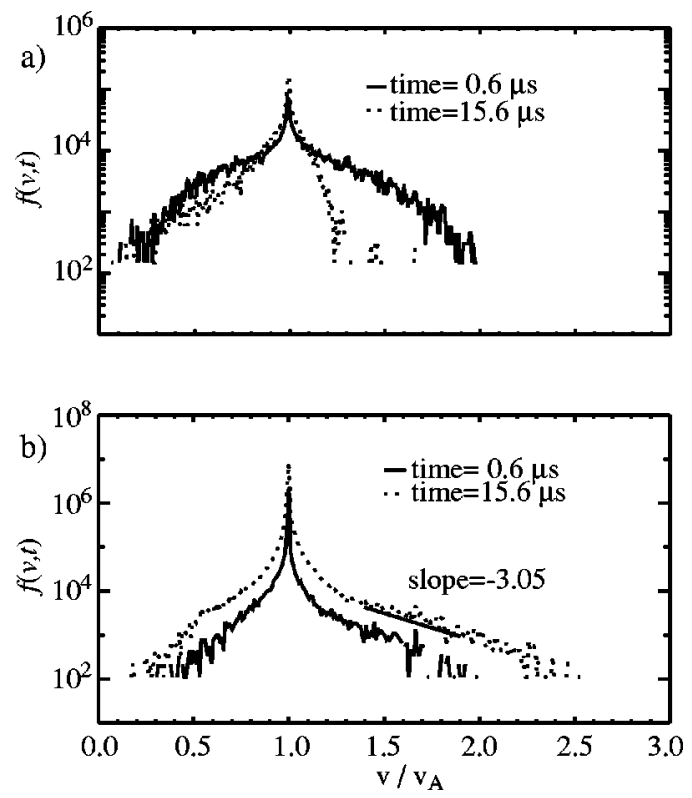

FIG. 10. "Tail formation (with particle resupply)." Distribution functions $f(v, t)$ of particles remaining within the simulation domain (top panel), and of particles that have escaped the domain (bottom panel), for a simulation in which particles are resupplied after escape (as described in the text), for two times $t=0.6 \mu \mathrm{s}$ and $t=15.6 \mu \mathrm{s}$. Note that in the top panel particles spread to both higher and lower speeds with a core distribution that resembles a Maxwellian centered about the initial speed, while in the bottom panel an exponential tail with the slope of -3.05 is observed.

are detected cumulatively in time outside the domain due to SSX-like acceleration? Figure 10 provides an answer to this question, showing speed distributions again at $t=0.6 \mu \mathrm{s}$ and at $t=15.6 \mu \mathrm{s}$. One can see that the escaped particles also have a broad distribution, with significant numbers of particles at speeds lower than $v_{A}$. From the Figure we can see that the higher speed particles seem to follow an exponential distribution fairly well. However, given the dynamic range of the speed, it is difficult to distinguish this fit from other possible functional forms. For example, the same speed distribution follows a power-law distribution (not shown) just about as well as it follows an exponential.

As a final comparison between the simulation and SSX data, we show, in Fig. 11 a sample of energetic particle pulses detected by SSX and by the TRIM particle simulations. The particle data are acquired using Retarding Grid Energy Analyzers (RGEA) with selected bias voltages. Ions above the gate energy are collected and contribute to the measured particle current. In the illustration, the gate voltages correspond to proton speeds of $\sqrt{2} V_{A}, \sqrt{3} V_{A}$, and $2 V_{A}$ (about 100, 150, and $200 \mathrm{~V}$, respectively). For the comparison, the escaping test particle data are analyzed with the same threshold energies. A time of flight delay has been subtracted from the timing of each experimental pulse, using a conservative estimate of $25 \mathrm{~cm}$ for the drift distance and (since the velocity distributions are steep) the threshold velocity as given by the gate energy. One can see that the generation of a burst of super-Alfvénic particles leaving the device in both cases. Also in both cases the pulse peak occurs a few microseconds $\left(\sim \tau_{A}\right)$ after the "zero" time defined by the peak of the reconnection zone magnetic energy. 

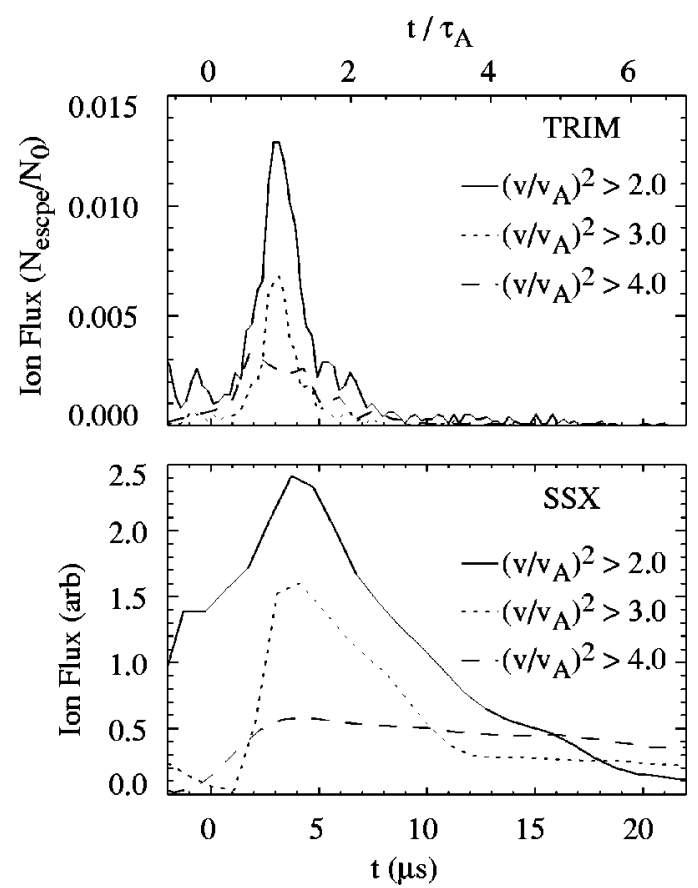

FIG. 11. Flux of protons/test particles with energies above $2 V_{A}^{2}, 3 V_{A}^{2}$, and $4 V_{A}^{2}$, from TRIM test particle simulation data (top) and from SSX data (bottom). Pulses of super-Alfvénic particles are observed in both cases, with the main pulses occurring several $\mu$ s after $t=0$, the time of peak magnetic energy in the reconnection zone.

These traces also portray some information about the particle spectra. Although the SSX pulses are broader, the nature and timing of the pulses appears to be very similar. This again reinforces the view that the TRIM simulations capture much of the basic physics of SSX.

\section{DISCUSSION}

We have examined the behavior of energetic charged particles in the Swarthmore Spheromak Experiment. Particular emphasis is placed on those that are accelerated near the interaction region between the two partially relaxed spheromak plasmas. The particles respond to electromagnetic fields that are modeled using an MHD simulation code. The MHD description of SSX behavior so obtained has been compared here and elsewhere with SSX experimental field and particle measurements. The principal results are as follows.

(1) MHD simulation shows that the interaction region is characterized by formation of magnetic X-points and colocated filamentation of electric current density; (2) there are two phases to the MHD evolution. First, there is an intense period of interaction as the two spheromak plasmas first encounter one another. Reconnection activity is strongly driven in this phase. Second, there is a longer period of milder MHD activity, which is characterized as either weakly driven or spontaneous reconnection; (3) the reconnection rate, measured by the locally averaged induced electric field, peaks strongly during the early period of activity. Later, the reconnection rate hovers around $1 / 10$ in dimensionless units, as expected from earlier work ${ }^{10,19}$ for either MHD or collisionless reconnection, under a broad variety of circumstances; (4) test particle distributions respond by broadening, with significant numbers of particles decelerated and accelerated; (5) for short periods of time (less than an Alfvén crossing time), particles see, on average, a nearly constant electric field, consistent with models of trapping and acceleration in turbulent reconnecting fields; ${ }^{11}$ (6) escaping particles on average have a higher energy than those remaining in the simulation domain; (7) the spectrum of accelerated particle is approximately exponential within the domain, and for the escaped particles it is either exponential or power law.

This description of MHD activity and particle acceleration has been developed by examining experimental data and results of both MHD and test particle simulation data. This appears to be a fruitful way to proceed, as each approach has distinct advantages as well as limitations. Correspondence between modeling and experiment allows for stronger conclusions to be drawn. While our description of reconnection and associated acceleration mechanisms cannot be considered complete, we believe that the present description is the first time that these elements have been brought together to describe charged particle behavior in a reconnection scenario. We again recall that kinetic effects, especially those associated with low collisionality and self-consistent effects of energetic particles, are expected to be influential in SSX and have not been included in the present modeling effort. Along with influence of the equation of state (also oversimplified here) these factors may be responsible for features of the onset of reconnection and acceleration, and structure of the reconnection and acceleration region, that are not accessible with our currect modeling approach. Clear directions for further research are suggested. As experimental diagnostics on SSX are improved, we expect to be able to examine more subtle issues pertaining to timing of the acceleration at various energies. Analysis of the spectral distribution and other statistical properties of MHD fluctuations is also underway, using improved probes. Improved MHD descriptions, including, for example, the Hall effect, should permit more refined and accurate treatment of the current filamentation process. It remains to be determined if this will substantially affect the characteristics of the acceleration mechanism. Finally, as we acquire greater confidence that our modeling efforts provide plausible physical interpretation of the SSX experimental device itself, we are becoming more interested in examining the scaling of observed reconnection and acceleration mechanisms to other physical parameters. We can, for example, envision larger SSX devices in the numerical models, and study the scaling of physical processes such as energy spectra, maximum energy, and other properties of the energetic particles. In this way, insights may be gained regarding similar processes that occur in much different parameter regimes on the sun and in astrophysical settings.

\section{ACKNOWLEDGMENTS}

This work was performed under Department of Energy (DOE) Grant Nos. DE-FG02-97ER54422 and DE-FG0298ER54490, and was also partially supported by the NSF under Grant No. ATM-9713595 at Bartol. M.R.B. is a DOE 
Junior Faculty Investigator. Special thanks to S. Palmer and D. Radcliff for assistance with the apparatus.

${ }^{1}$ C. G. R. Geddes, T. W. Kornack, and M. R. Brown, Phys. Plasmas 5, 1027 (1998).

${ }^{2}$ T. W. Kornack, P. K. Sollins, and M. R. Brown, Phys. Rev. E 58, R36 (1998).

${ }^{3}$ V. S. Lukin, G. Qin, W. H. Matthaeus, and M. R. Brown, Phys. Plasmas 8, 1600 (2001)

${ }^{4}$ M. R. Brown, Phys. Plasmas 6, 1717 (1999).

${ }^{5}$ D. D. Schnack, I. Lottati, Z. Mikic, and P. Satyanarayana, J. Comput. Phys. 140, 71 (1998).

${ }^{6}$ R. G. Giovanelli, Mon. Not. R. Astron. Soc. 107, 338 (1947).

${ }^{7}$ J. W. Dungey, Cosmic Electrodynamics (Cambridge University Press, Cambridge, 1958), pp. 98-102.

${ }^{8}$ P. A. Sweet, Nuovo Cimento, Suppl. 8, 188 (1958).

${ }^{9}$ E. N. Parker, Astrophys. J. 123, 644 (1958).

${ }^{10}$ W. H. Matthaeus and S. L. Lamkin, Phys. Fluids 29, 2513 (1986).

${ }^{11}$ J. J. Ambrosiano, W. H. Matthaeus, M. Goldstein, and D. Plante, J. Geophys. Res. 93, 14383 (1988)
${ }^{12} \mathrm{P}$. Gray and W. H. Matthaeus, Particle Acceleration in Cosmic Plasmas, AIP Conference Proceedings 264, Newark, Delaware, 1991, edited by G. P. Zank and T. K. Gaisser (AIP Press, New York, 1992), p. 261.

${ }^{13}$ T. Kobak and M. Ostrowski, Mon. Not. R. Astron. Soc. 317, 973 (2000).

${ }^{14}$ A. R. Bell, Mon. Not. R. Astron. Soc. 182, 147 (1978); 182, 443 (1978).

${ }^{15}$ R. D. Blandford and J. P. Ostriker, Astrophys. J. Lett. 221, L29 (1978).

${ }^{16}$ F. C. Jones and D. C. Ellison, Space Sci. Rev. 58, 259 (1991).

${ }^{17}$ M. L. Goldstein, W. H. Matthaeus, and J. J. Ambrosiano, Geophys. Res. Lett. 13, 205 (1986).

${ }^{18}$ E. N. Parker, Cosmical Magnetic Fields: their Origin and Activity (Oxford University Press, New York, 1979), p. 392.

${ }^{19}$ M. A. Shay, J. F. Drake, R. E. Denton, and D. Biskamp, J. Geophys. Res. 103, 9165 (1998).

${ }^{20}$ M. A. Shay, J. F. Drake, B. N. Rogers, and R. E. Denton, "Alfvénic collisionless magnetic reconnection and the Hall term," J. Geophys. Res. 106, 3759 (2001).

${ }^{21}$ X. G. Wang, A. Bhattacharjee, and Z. W. Ma, J. Geophys. Res. 105, 27633 (2000).

${ }^{22}$ W. H. Matthaeus, J. J. Ambrosiano, and M. L. Goldstein, Phys. Rev. Lett. 53, 1449 (1984) 